\title{
THE CHARACTER OF THE OPTICAL VARIABILITY FOR THE BL LACERTAE OBJECTS OJ 287
}

\author{
H. Richard Miller and Mi chael T. Carini \\ Department of Physics and Astronomy \\ Georgia State University, At lanta, Georgia 30303
}

The BL Lacertae objects, OJ 287 , has long been known to exhibit large amplitude variations on time scales as short as a few days. The time rate of change in brightness for these variations has been observed to be in excess of $1.0 \mathrm{mag}$. per day on several occasions. However, the nature of the optical variations on time scales significantly shorter than a day has not been well established, and in many cases the results have been controversial (Carrasco et al, 1985, Lawrence et al 1987, McHardy and Czerny, 1987). In order to address this problem, we have obtained observations of OJ 287 on four different nights during the past two years using the KPNO 0.9 meter telescope equipped with the direct OCD camera. Variations were detected for OJ 287 on each night with ranges of $0.05 \mathrm{mag}$. to $0.10 \mathrm{mag}$. The variations detected on 1986 November 9 are typical of those observed on each of the nights and is shown in Figure 1. A power spectrum analysis of the data was performed and no convincing evidence for the presence of any periodicity was found. Based on this analysis, the short term optical variations observed for OJ 287 have a very different character than the rapid $x$-ray flickering observed for Seyfert galaxies which have been characterized by $1 / \mathrm{f}$ - noise. Therefore, these observations would imply sizes for the source region $3 \times 10^{-5} \mathrm{pc}$. If one assures that the underlying engine is a supermassive blackhole, then the time scales of the variability suggests masses for the blackhole of $10^{7} \mathrm{M}_{0}-108 \mathrm{M}_{0}$.

\section{References}

Carrasco, L., Dultzin-Hacyan, D., and Cruz-Gongalez, I . 1985, Nature, $314,146$.

Lawrence, A., Watson, M. G., Pounds, K. A., and Elvis, M. 1987, Nature, 325,694 .

McHardy, I., and Czerny, B. 1987, Nature, 325, 696 . 


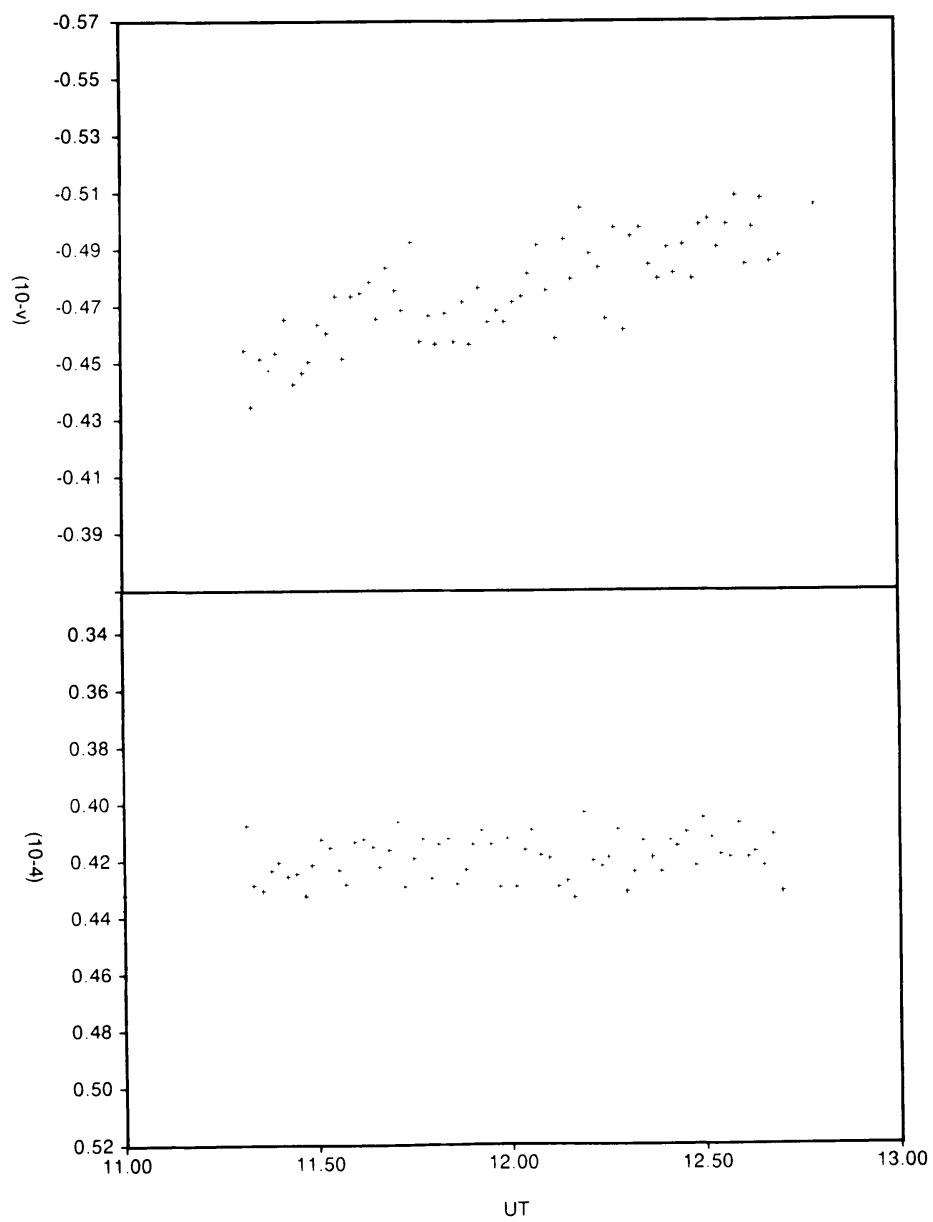

Figure 1. The differences in the optical variations of OJ 287 and comparison star 10 observed on 1986 November 9 are plotted in the upper panel. The variations of the differences of comparison star 10 and comparison star 4 are plotted in the lower panel. 